https://doi.org/10.31713/m1125

\title{
METHOD OF CALCULATING THE STRESS-STRAIN STATE OF THE DEPTH WELL AND AREA AROUND THE WELL
}

\author{
Mykhailovska O.V.
}

\author{
Ph.D., Senior Researcher, Associate Professor of the Department \\ of Oil and Gas Engineering and Technology, National University \\ «Yuri Kondratyuk Poltava Polytechnic», Ukraine
}

\begin{abstract}
A mathematical model was used to solve the problem of estimating the stressstrain state of an array near a vertical well of great depth. The model includes equations of equilibrium, dependences between components of deformations and components of displacements, equations of properties of the environment, intensity of stresses and intensity of deformations, and also boundary conditions.

To derive the calculated dependences, an elementary cylinder with a hole is considered. Cylinder of constant radius. The elementary cylinder of constant thickness is loaded with internal and external pressure. It is determined that for operative decision-making related to various problems arising in the process of construction of deep wells (oil, gas, etc.), their operation, as well as in their design, very relevant analytical studies based on the use of engineering method assessment of the stress-strain state of the deep wellbore.

It is determined that the results of the application of the proposed method are the rapid assessment of the stress state of the rocks of the shaft, near the wellbore and in the array. The result is a qualitative assessment of the possible distortion of the productivity of the formations before their tests and scientific substantiation of the parameters of the technological process that prevent possible complications in the drilling of deep wells. Recommendations for the use of this method are given.
\end{abstract}

\section{Introduction}

Most of the complications in wells (absorption, landslides, etc.) are the result of a certain stress state of the array with all its internal processes that took place before and are now. Without establishing and evaluating the stress-strain state of the array around the well, identifying its nature and mechanism of occurrence. In this case, it is quite difficult to take scientifically sound measures to prevent complications when drilling wells.

Modern technologies allow to effectively drill wells at elevated temperatures, formation pressures. To calculate the parameters of such technologies, data on the stress state of rocks are needed, both 
along the wellbore and in the wellbore zone. It is also necessary to study formation pressure and rupture pressure of rocks in wells and on the surface - in identical laboratory conditions. Modern deep wells are drilled using the same techniques and the same technological processes as at shallow depths (up to 2.5-3.0 km). However, the drilling speed in the same rocks is much lower, and the accident rate and complexity in wells is higher. The complexity of dealing with complications in deep wells is also higher [1].

There are many methods for determining the stress-strain state of wells during drilling using analytical expressions, which became the basis of the mathematical apparatus of software packages. Numerical methods are currently the main methods of solving mathematical problems. They are characterized by reducing the process of solving a mathematical problem to a finite sequence of operations on numbers. They lead to results presented in the form of numbers, numerical vectors and matrices, numerical tables and so on. Their value is being clarified in parallel with the development of computer technology [8].

At the same time, the results obtained by numerical methods usually contain errors, being only approximations to the required answers. This is due to a number of objective reasons, among which are not directly related to calculation methods. Therefore, there is a need for preliminary rapid assessment of the state of the deep well using engineering techniques.

\section{Basic investigation}

2.1 Analysis of the features of the stress - strain state of the shaft and the shaft zone of a deep well

Analysis of drilling wells in the areas showed that the most common complications are due to the violation of the stability of the well walls. For this reason, sometimes wells do not reach design depths and must be eliminated. They do not fulfill their purpose at the expense of their construction of large funds. In order to reliably assess the stress-strain state of wells, it is necessary to substantiate the integral parameter (crack opening pressure), which characterizes the state of rocks in terms of their occurrence in the well. Rocks near the well are exposed to three main pressures: gravitational $P_{g}$, lateral geostatic $P_{2}$, and well back pressure $P_{1}$. The array pressure from the 
wellbore consists of the hydrostatic pressure of the drilling fluid column and the hydrodynamic pressure due to the technological processes in the well.

Hydrodynamic pressure (usually an order of magnitude less than hydrostatic) plays an indirect role in the occurrence of barrel deformations. It accelerates the time of destruction, ie is not the cause. The determinants are the pressures $P_{1}, P_{2}, P_{\mathrm{g}}$, [11,12]. According to modern ideas, the main factors influencing the stability of well walls are the stress state of rocks and physico-chemical interaction of rocks with drilling fluid. The direction of physicochemical processes in the system "formation - well" is determined by the chemical potential of the drilling fluid and the chemical potential of rocks. In the general case, involuntary thermodynamic processes are directed towards equalization of potentials. One of the methods of influencing the formation is the use of inhibited and mineralized solutions. Inhibition, as a rule, is achieved by mineralization of the solution, and in parallel with this are regulated and osmotic processes. Additives are introduced into the solutions to ensure saturation of their ions in the well: $\mathrm{K}^{+} ; \mathrm{Ca}^{2+}$, $\mathrm{Mg}^{2+}$ and $\mathrm{Na}^{+}$.

The use of inhibited solutions in wells prone to deformation, gave positive results [10]. And since osmotic processes are regulated along with inhibition, it can be assumed that the osmosis process, along with other factors, has a certain effect on the stability of well walls. The practice of drilling has shown that in some cases weighting of drilling mud without its chemical treatment has not solved the problem of stability of the wellbore. At the same time, it has not yet been possible to completely solve the problem of eliminating complications and increasing the stability of well walls when drilling in unstable rocks by the method of inhibition. As a result of physicochemical action of the solution on the formation, it is possible to achieve the stability of the wellbore only in conditions of stress close to equilibrium. In this case, the osmotic pressures, the value of which is at best 1-3 MPa, can only compensate for the hydrodynamic pressures in the well.

Thus, the main factor determining the stability of the wellbore is its stress state. It depends on the magnitude of external influences $\left(P_{1}, P_{2}, P_{\mathrm{g}}\right)$, formation fluid pressure and physical and mechanical 
properties of rocks in the conditions of their occurrence. Predicting the current stresses in rock massifs is of great practical importance. These components together with physical and mechanical and rheological properties of rocks serve as a basis at calculation of stability of walls of not fixed trunks of wells and calculations on durability of casings. Also, these components are the basis for assessing the possible distortion of the productivity of formations before testing, the rationality of the adopted scheme of destruction of rocks at the bottom and the reliability of separation of productive formations from aquifers after casing casing.

Based on the analysis of the results of research [3,4], it was found that for the stability of the wellbore walls in any case (any rock) it is necessary to strive to obtain in the well conditions close to equilibrium. Most of the complications that currently occur in the practice of drilling are due to non-compliance with this requirement. To eliminate this circumstance, the method of engineering calculation of the stress-strain state of the array can be used. With which it is possible to quickly and reliably establish the stress state of the rocks that make up the walls of the well, near the well and in the depth of the massif and make objective decisions under given conditions. Known methods for estimating the stress state of an array of rocks: using analytical calculations and methods of mechanical and mathematical modeling.

The reliability of analytical calculations is determined by the degree of conformity of the mathematical model to the natural state. And also depends on how accurately the indicators of mechanical properties characterize the behavior of the rock in a given stress state of the array. The greatest relevance, in connection with the efficiency of calculations of stresses and displacements are engineering methods of calculation. They, in turn, are the most complex because they require sufficient accuracy of the calculated information.

Compared with analytical methods for studying the stability of the wellbore as a function of the stress-strain state of rocks, mechanical modeling has a number of advantages. One of them is the possibility of further complicating the model to achieve greater compliance with the natural sample. That is why mechanical modeling in the study of the stability of the wellbore and wellbore is often the main. However, the complexity of the studied processes 
leads to the complexity of the equipment to be used and the complexity of the experiments. The results of experiments, in most cases, can be interpreted and used only for qualitative evaluation of the studied process. For this reason, the analytical method of estimating the stress state of an elastic - plastic array with a vertical deep well was used.

\subsection{Current trends in the method of calculating the stress- strain state of a deep wellbore}

The condition of the problem is formulated as follows. We have a heavy array bounded on top by a horizontal plane, from the plane to the depth of the array (Fig. 1) is a vertical well. The elastic array is in equilibrium under the action of its own weight, no other effort is applied to the array. You need to find the voltage in such a weakened well array. Instead of an elastic half-space with a cylindrical well of finite depth, a cylinder of radius $r_{2}$ with a vertical cavity $r_{1}$ is usually considered. The influence of end effects (wellhead and bottom of the well) is not taken into account, because the depth of the well is many times its diameter. As a result, the dependence of normal stresses $\sigma_{r}$, $\sigma_{t}$, and displacements $u$ on the radius of the cylinder $r_{1}, r_{2}$, stresses and displacements must be inseparable and unambiguous functions $r$ and $z$ inside the array.

To date, the solution of the problem, in the formulated formulation, is used for engineering assessment of the stress state of rocks in the wellbore zone. As we approach the well walls, the normal radial stresses $\sigma_{\mathrm{r}}$ decrease (in absolute value), and the normal tangential stresses $\sigma_{\mathrm{t}}$ increase. Vertical normal stresses $\sigma_{\mathrm{z}}$ do not depend on the distance to the well walls, but remain constant and are determined by the value of geostatic pressure. However, along with experimental studies, a significant difference from the above stress distribution was found. Therefore, to establish the pattern of stress distribution in the shaft zone of a deep well, the task was to study the stress state of this zone of the well drilled in the formation, subject to gravitational forces and back pressure (Fig. 1).

The real relationship between stresses and strains for rocks is curvilinear and plastic deformations often appear immediately after loading. 


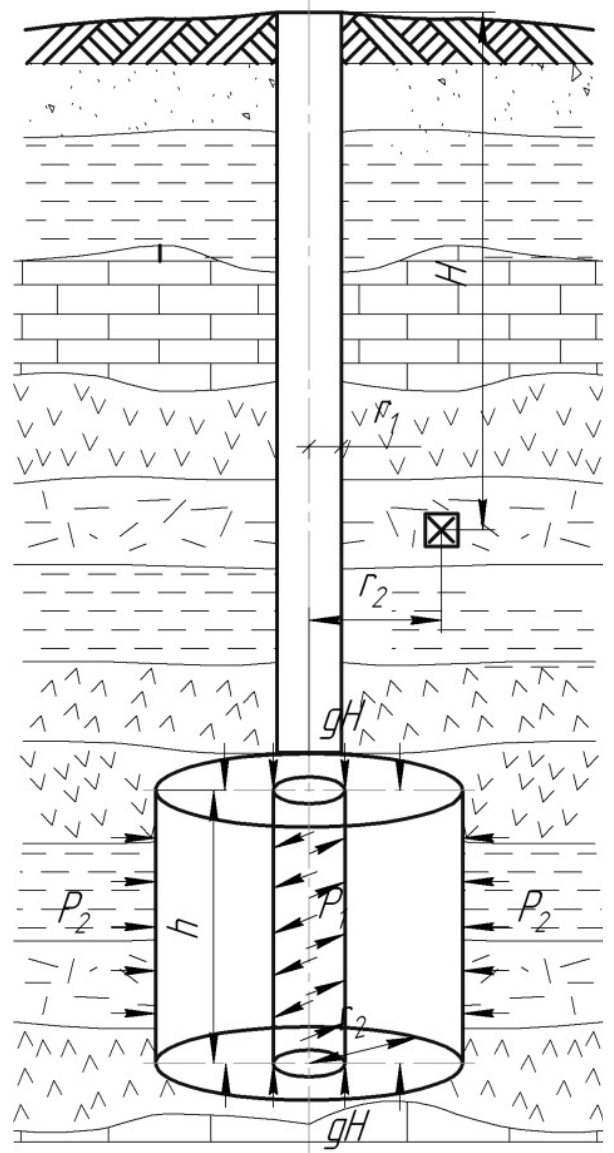

Fig. 1. Calculation scheme

Therefore, it is necessary to introduce the concept of the modulus of deformation $E_{0}$, equal to the ratio of the normal stress to the total relative longitudinal deformation, including residual: $E_{0}=\sigma / \varepsilon_{\Pi}$

The magnitude of the modulus of elasticity for the same rock sample is slightly larger than the modulus of deformation $\left(E>E_{0}\right)$. and the angle of internal friction $\rho$. Among the characteristics of strength, the tensile strength of uniaxial compression is of particular importance. This is the main constant of various materials, including rocks. Practically all geomechanical researches begin with definition of a limit of durability on uniaxial compression of samples of rocks. 
The study began with the determination of the tensile strength $\sigma_{\mathrm{T}}$ by the parameter - fracturing stresses $\sigma_{\mathrm{gr}}$. In the study of samples, the tensile strength is set by the load when the failure of the sample and the tensile strength of uniaxial compression is defined as the ratio of the destructive force $P$ to the cross-sectional area of the sample $F$, ie, MPa

$$
R_{c}=P / F \text {. }
$$

However, there are external and internal reasons that contribute to the fact that the field of stresses and strains in the rock sample will be heterogeneous. Studies show that for the same lithological difference, different test conditions can change the strength limit to uniaxial compression up to 5 times. Internal causes that lead to a violation of the homogeneity of the field of stresses and strains in the natural structural heterogeneity of rocks (latent fracture, the inclusion of different sizes and different strengths, pores, etc.). It is impossible to obtain a perfectly homogeneous field of stresses and strains in the formation according to the sample. It is possible to obtain a state close to homogeneous. It can be assumed that the uniaxial compressive strength of the rock samples will always be higher than the strength of the rock mass. It is established that the problem of linear heredity can be formally considered as a problem of the theory of elasticity, in which instead of elastic constants $E$ and $\mu$ it is necessary to use their temporary analogues (integral operators) $-\bar{E}, \bar{\mu}$.

The difficulty of solving problems by methods of hereditary creep theory is to decipher temporary operators. Often, when the boundary conditions are constant, the method of variable modules is used to solve geomechanical problems, which consists in using time functions $E(t)$ and $\mu(t)$ instead of integral operators, which can be found by the formulas

$$
\begin{gathered}
E(t)=E /(1+\Phi) \\
\varepsilon(t)=\frac{\sigma_{0}}{E}\left(1+\frac{\delta t^{1-\alpha}}{1-\alpha}\right)=\frac{\sigma_{0}}{\bar{E}} ; \\
\Phi=\frac{\delta t^{1-\alpha}}{1-\alpha}
\end{gathered}
$$


where $\delta$ and $\alpha$ are the rheological characteristics obtained experimentally; $F$ is the creep function. The time function for the Poisson's ratio is defined by the expression $\mu(t)=0,5-\frac{0,5-\mu}{1+\Phi}$.

It should be noted that the use of the theory of hereditary creep is justified only if the operating stresses do not exceed the long-term strength of the array. The mechanical properties of rocks obtained in the laboratory do not correspond to the mechanical properties of real rock massifs and this discrepancy manifests itself in the form of a large-scale effect. The sample of rocks can be said as some idealized model of the rock mass. This circumstance became the reason for the search for mechanical constants in kind, ie directly in the reservoirs (Fig. 1).

According to the hypothesis of homogeneity of the rock mass, we select the space around an arbitrarily chosen point of elementary volume in the form of a cube (Fig. 2). When the rock is deformed, internal forces $\left(P_{x} \cdot P y \cdot P_{2}=\gamma H\right)$ are applied to the faces of the cube, the intensity of which is stresses. In the general case, the stresses form an angle $\alpha$ with the normal drawn to the face of the cube. For ease of consideration, they can be decomposed into normal and tangent components (Fig. 2).

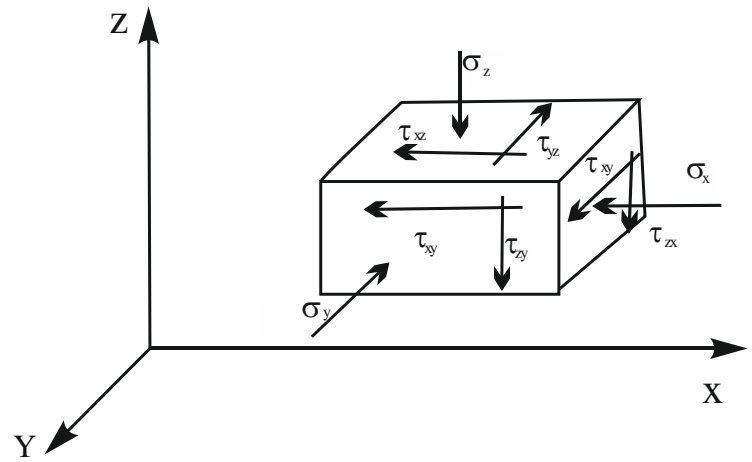

Fig. 2. Estimated stress scheme in the rock sample according to the hypothesis of array homogeneity

These components are called stress components. Thus, there are three components of normal $\sigma_{x}, \sigma_{y}$ and $\sigma_{z}$ and six - tangential stresses - $\tau_{x y}, \tau_{y x}, \tau_{x z}, \tau_{z x}, \tau_{y z}, \tau_{z y}$. For the convenience of analysis of the stressstrain state introduce the concept of average or geostatic stress. $\sigma_{0}=3^{-1}\left(\sigma_{x}+\sigma_{y}+\sigma_{z}\right)$. By analogy with the stresses can be introduced 
into the concept of average deformation: $\varepsilon_{c p}=\frac{1}{3}\left(\varepsilon_{x}+\varepsilon_{y}+\varepsilon_{z}\right)$. The coordinate system can always be changed so that the direction of the coordinate axes coincides with the lines of action of such normal stresses at which the tangential stresses are zero. These stresses are called principal and denote $\sigma_{1}, \sigma_{2}$ and $\sigma_{3}$. And $\sigma_{1}>\sigma_{2}>\sigma_{3}$. Under the action of stresses applied to the faces of the elementary cube, it is deformed.

Each of its faces will change linear and angular dimensions. Linear changes are estimated by the components of displacements $y, v, w$ and linear relative deformations $\varepsilon_{x}, \varepsilon_{y}, \varepsilon_{z}$, and angular - by angular deformations $\tau_{x y}, \tau_{y x}, \tau_{x z}, \tau_{z x}, \tau_{y z}, \tau_{z y}$ etc.

Mechanical failure due to force can be defined as the result of some deformation and rupture of structural connections of the structural material. The study of this process, as well as any problem, is based on physical and mathematical models that mimic real solids. The results of their research on the laxative effect of microdefects on the strength of rocks A.A. Griffiths embodied the theory of strength. The theory formed the basis of further research in the field of socalled microdefect strength theories. The criteria used to determine whether the destruction of the rock will be

$$
\begin{aligned}
& \left(\sigma_{1}-\sigma_{2}\right)+8 R_{p}\left(\sigma_{3}-\sigma_{1}\right)=0 \text { if } 3 \sigma_{3}+\sigma_{1}>0, \\
& \sigma_{3}=R_{p}, \text { if } 3 \sigma_{3}+\sigma_{1}<0 .
\end{aligned}
$$

Accepting in the first expression $\sigma_{3}=0$, we obtain the ratio between the tensile strength of uniaxial compression and the tensile strength of uniaxial tensile

$$
R_{\mathrm{c}}=-8 R_{p},
$$

which is fully consistent with the research data for rocks. Microdefective theories are unique in that they consider fracture as the result of disintegration of a solid body along a single trunk crack. On the walls of the well brittle destruction is a little different, it is realized in the form of a system of cracks. To describe such a mechanism of crack formation, it is necessary to introduce special preconditions into working hypotheses. When deforming solids, there are always two mechanisms by which the material is destroyed - plastic flow and brittle fracture (cracking, tearing). The concept of brittle and viscous fracture is the physical basis for the development of certain cri- 
teria of strength. If the plastic deformation caused by tangential stresses loosens the material and prepares it for rupture, the violation of continuity occurs under the action of normal tensile stresses. In solids such as rocks, plastic deformations begin at relatively low loads. Among the many chaotically arranged crystals, there will always be a number of the least favorably oriented in relation to external forces and having internal defects such as dislocations. These crystals are deformed plastically even with relatively small external forces. The number of these crystals is relatively small and local plastic deformations do not significantly affect the overall relationship between force and displacement, which are inherent in the initial stage of loading. With high external forces, plastic deformations become predominant. According to the criteria of Moore's theory, the fracture process in the local region of a solid body, the tangential stresses of which are quite fully characterized by the intensity of stresses and loosen the material by shear. Under the action of normal stresses $\sigma$ cracks open. The combined action of two types of destruction (shear and separation) leads to a loss of strength of the rock. Such a scheme of destruction is consistent with dislocation and dilaton theories of brittle cracks.

According to Mora's hypothesis, we can assume that the strength of the rock practically depends only on the members of this expression. That is, determines the time of transition of the rock to a plastic state.

$$
\sigma_{i}=\frac{\sqrt{2}}{2} \sqrt{\left(\sigma_{1}-\sigma_{2}\right)^{2}+\left(\sigma_{2}-\sigma_{3}\right)^{2}+\left(\sigma_{3}-\sigma_{1}\right)^{2}},
$$

Theories of rock pressure to determine the load on the casing in relation to deep wells are characterized by three directions.

The first direction is based on the use of simple physical models, according to which the load on the walls of wells is created by lateral rocks that have some degree of destruction. The magnitude of the load does not depend on the time, technology and characteristics of the casing. The calculation methods of this direction are based on the theory of calculation of retaining walls.

The second direction is based on the hypotheses of the interaction of casing, which are deformed together with the surrounding rock mass. In this case, the load on the pipes depends on the rigidity of the casing and the parameters of the technology of its construction. 
The third direction is formed by empirical methods, which are based on the results of properly processed and presented results of laboratory experiments. The pressure on the casing in the wellbore occurs in rocks of any strength and at any depth in proportion to this depth. However, practice shows that when crossing strong rocks, the walls of the well can remain stable for a long time, even without casing.

The condition is expected to be fulfilled

$$
\left[\sigma_{T}\right] \geq \frac{2 \mu}{1-\mu} \gamma H
$$

where $\left[\sigma_{T}\right]$ - the strength limit of rocks in terms of its occurrence.

In the first approximation, the following dependences were used to analyze the stress state of an elastic massif with a hole (for calculations of the stress state of an element (Fig. 2) of a wellbore) $[1,12]$. In the case of vertically acting geostatic pressure of rocks - $\gamma H$. If there are horizontal forces of the lateral strut and (in the general case, they are equal to each other) $-P_{x}=P_{y}=P_{2}$.

In cases where $\mu=0$ and, and at $\mu=0.25$, at $\mu=0.5$ already, ie always less than $\gamma H$. In the case of a well filled with liquid $\mathbf{P}_{1}$, it will be equal to the hydrostatic pressure.

To solve this problem Dynnyk A.N, and Tymoshenko S.P. [9, 10] recommend calculation dependences that allow to find on the basis of the known Lamé solution for a thick-walled cylinder loaded with external and internal distributed load. Makarov L.V. special studies were conducted to verify the validity (scope) of the formulas proposed by Dynnyk A.N, Tymoshenko S.P. and Lekhnitsky S.G. For research he used generalized equilibrium equations of an elastic body in cylindrical coordinates

$$
\begin{gathered}
(1-\mu)\left[\frac{1}{r} \frac{\delta u}{\delta r}-\frac{u}{r^{2}}+\frac{\delta^{2} u}{\delta r^{2}}\right]+\frac{1-2 \mu}{2} \frac{\delta^{2} u}{\delta z^{2}}+\frac{1}{2} \frac{\delta^{2} w}{\delta z \cdot \delta r}=0 \\
\frac{1}{2}\left[\frac{1}{r} \frac{\delta u}{\delta z}+\frac{\delta^{2} u}{\delta z \cdot \delta r}\right]+\frac{1-2 \mu}{2}\left[\frac{1}{r} \frac{\delta w}{\delta r}+\frac{\delta^{2} w}{\delta r^{2}}\right]+(1-\mu) \frac{\delta^{2} w}{\delta z^{2}}=k g \frac{(1+\mu)(1-2 \mu)}{E}
\end{gathered}
$$

The components of the stress tensor in this problem are found in the form

$$
\begin{gathered}
\sigma_{r}=\lambda\left(\frac{u}{r}+\frac{\delta w}{\delta z}\right)+(\lambda+2 \bar{\mu}) \frac{\delta u}{\delta r} ; \sigma_{t}=\lambda\left(\frac{\delta u}{\delta r}+\frac{\delta w}{\delta z}\right)+(\lambda+2 \bar{\mu}) \frac{u}{r} \\
\sigma_{z}=\lambda\left(\frac{\delta u}{\delta r}+\frac{u}{r}\right)+(\lambda+2 \bar{\mu}) \frac{\delta w}{\delta z}
\end{gathered}
$$


Where

$$
\begin{aligned}
& \lambda=\frac{\mu E}{(1+\mu)(1-2 \mu)} ; \bar{\mu}=\frac{E}{2(1+\mu)}: \\
& \tau_{r t}=\tau_{t z}=0 ; \tau_{r z}=\bar{\mu}\left(\frac{\delta u}{\delta z}+\frac{\delta w}{\delta r}\right) .
\end{aligned}
$$

Boundary conditions

$$
\begin{array}{lll}
\text { for } z=h & \sigma_{z}=0 ; & \tau_{z r}=0 ; \\
\text { for } z=0 & u=0 ; & w=0 ; \\
\text { for } r=r_{1} & \sigma_{r}=0 ; & \tau_{r z}=0 ; \\
\text { only when } P_{1}=0 u & r=r_{2}, & u=0, \\
& \text { a } \sigma_{z}=-\frac{\mu}{1-\mu} g(h-Z) .
\end{array}
$$

For the accepted equations, the components of the stress tensor were found numerically using the grid method and replacing the equations in the derivatives with the equations of differences. Computers were used to solve these equations. As a result of the study it was found: 1) Vertical normal stresses decrease in absolute value as you approach the walls of the well, the maximum reduction in the main calculated version was $5 \%$ 2) The decrease is due to the protrusion of rocks towards the well. With increasing $\mu$ there is a more intense protrusion. Subsequently, the same conditions with a mathematical description were extended to the elastic-plastic region. The problem in the new interpretation was solved first in an elastic formulation.

The values were found $\sigma_{i}$ and compared with the value accepted for a particular material on the basis of experimental data. If, $\sigma_{i}<\sigma_{i n p}$ then it was considered that the solution describes the elastic deformation, if, $\sigma_{i}>\sigma_{i n p}$ then it is a plastic region. The solution was made with additional, in addition to the boundary conditions of the problem and assumptions for the adopted method.

According to Makarov L.V. $\sigma_{i}=\phi\left(\varepsilon_{i}\right)=3 Y\left(1-w_{i}\right) \varepsilon_{i} ; \sigma_{i}<\sigma_{i n p}-$ elastic area; $\sigma_{i}>\sigma_{i n p}$ - not an elastic area. $Y$ - modulus of elasticity of the second kind; $w_{i}$ - some function, $w_{i}=w_{i}\left(\varepsilon_{i}\right)$, which varies within the region in the region of plastic deformations and is equal to 0 in the elastic region; $\sigma_{i}, \varepsilon_{i}$ - intensity $\sigma$ and deformations $\varepsilon$.

Conclusions based on the results of research were reduced to conclusions based on the solution of the same problem in an elastic formulation. As you approach the walls of the well in any of the hori- 
zontal planes, the stresses increase in absolute value, and the stresses decrease. This conclusion coincides with the currently widespread statement, including the results of research conducted by the author. The above shows once again that the task - to create an engineering method of calculation near the well drilled in the elastic-plastic massif, is quite difficult. This problem is difficult to solve even with the help of a computer, if the decision is made insufficiently sound initial equations and prerequisites for their solution.

The solution of the problem in this production was carried out like Makarov LV J.S. Erzhanov [5,6]. The difference of his decision is that Erzhanov Zh.S. instead of the grid method, he used the time step method. During the step-by-step transition, the stress-strain state was assumed to be altered due to the deformation of the body boundaries and the presence of physical nonlinearity. A computer is used to solve the system of equations. This allows to obtain the stress state of the array, to calculate the creep and relaxation and at the same time to compare the calculated and experimental data. Erzhanov's decision Zh.S. obtained in a fairly accurate form. The most acceptable solution for engineering practice is the one proposed by Yu.M. Lieberman $[9,10]$. He solved the elastic-plastic problem with respect to an unloaded massif composed of homogeneous, isotropic rock, in which the stress components are the same in all directions and are determined by the value before production $\gamma H$.

In deriving the calculated dependences, the condition of the boundary state of rocks is accepted, as which the hyperbolic curve from the origin is used (ie the solution is proposed in hyperbolic functions). This curve describes a material that is not able to withstand tensile strength, but partially retains compressive strength. However, recommended by Lieberman Yu.M. dependencies for calculations are in conflict with the accepted condition of the limit state. In them, the main value that characterizes the rock is $\sigma_{D}$ - the strength of the rock in the array in tension.

As a source Lieberman Yu.M. the equation of equilibrium, elementary volume in polar coordinates is accepted $\frac{d \sigma_{r}^{n n}}{d r}+\frac{d \sigma_{r}^{n \pi}-d \sigma_{r}^{n n}}{r}=0$, after substituting the values of stresses describing the condition of the limit state in the form of a hyperbolic 
curve, the basic differential equation is derived. $2 S h 2 \xi \frac{d \xi}{d r}-2 \sin \eta C h \xi \frac{d \xi}{d r}-2 \sin \eta \frac{S h 2 \xi}{r}=0$

The solution of this equation is obtained implicitly under the following boundary conditions:

1 - Stresses act on the well circuit $\sigma_{\mathrm{r}}=-P_{1}$.

2 - At the boundary between the region of inelastic deformations and intact array, the equality of stresses is observed: $\sigma_{r}^{n n}=\sigma_{r}^{y n}$ and $\sigma_{t}^{n n}=\sigma_{t}^{y n}$ or $\sigma_{t}^{n n}+\sigma_{r}^{n n}=\sigma_{t}^{y n}+\sigma_{r}^{y n}=2 \gamma H$.

The derived formulas determine the radius of the region of inelastic deformations and the stress distribution in the inelastic and elastic zones depending on the strength of the rock properties, the size of the production $r$, the depth from the surface $\mathrm{H}$ and the reaction of production P1. Despite the complexity of the mathematical description, these formulas can be used for engineering analysis of the problem. In such a setting, attempts were made to solve the problem of D.V. Danish, E.G. Leonov, I.K. Matrosov, Yu.P. Yellow, N.I Yagubov and others [8]. N.I. Yagubov in describing the behavior of the stump zone used viscoelastic models of Alpheus with the Laplace transform, as a result he obtained complex computational dependencies, the use of which in engineering calculations is quite complex.

Other of these authors used as mechanical characteristics of rocks: the coefficient of displacement of the rock in the region of inelastic deformations, the angle of internal friction of the rock, yield strength and compressive strength, the values of which are determined by the core. Given that the data obtained on the core do not always correspond to reality, because the interaction of rock with drilling mud and unloading of the core from rock pressure will cause serious distortions in the value of mechanical properties of the rock. Also, such indicators as compressive strength, coefficient of adhesion, internal friction angle and dynamic viscosity change during the transition of the rock to a plastic state, the solution becomes uncertain, does not meet the requirements of engineering practice. The study of the problems of analysis of the stress-strain state of deep wells is devoted to the work of V.I. Kareva, E.I. Shemyakina, I.L. Chernyaka, I.K. Fomenko, A.M. Papushi, E.M. Baranovsky, V.M. Stasenka [1-12]. Today there are a number of solutions taking into 
account certain properties of the array (anisotropy, inhomogeneity, time factor, etc.). The problems of drilling deep exploration wells in the Dnieper-Donetsk basin are that quite often there are situations when it becomes technically impossible and economically unprofitable to continue drilling. Based on a significant sample of factual data, it is investigated that complications and accidents, the elimination of which requires significant material costs, caused primarily by the manifestations of the destruction of the mountain massif, and occur mainly due to its imbalance. Virtually none of the wells over $5,500 \mathrm{~m}$ deep were drilled without complications and accidents.

Due to the uncertainty of the actual current stress-strain state and its failure to take into account when normalizing the density of flushing fluids, both in the design and drilling of wells, the mountain massif is always unbalanced. It is always heterogeneous in its structure and properties of rocks. The stress-strain state of rocks in the massif in the same stratigraphic horizons can have different values, which at the stage of development of drilling projects is impossible to predict. Prediction of current stresses in rock massifs is of great practical importance, because these components, together with the physicomechanical and rheological properties of rocks are the basis for calculating the stability of the walls of unfixed wells. Appropriate calculations are performed when assessing the productivity of formations before the tests, substantiation of the rationality of the adopted scheme of destruction of rocks at the bottom. Also, such calculations are necessary to determine the reliability of the isolation of productive strata from aquifers after cementing the casing. The main factor determining the stability of the wellbore is its stress state depending on the magnitude of external influences, reservoir fluid pressure and physical and mechanical properties of rocks under conditions of their occurrence.

Methods of mechanics of deformable media, in particular the theory of elasticity, plasticity, creep, etc., are widely used in the study of the stress-strain state of an array that has not lost its continuity. The scientific bases of the analysis of the stress-strain state of rocks are contained in the works of O.K. Angelopoulou, A.A. Gaivoronsky, V.D. Gorodnov, X. Feket, B.S. Filatov, L.A. Schreiner, R.M. Eigeles, R.S. Yaremiychuk and others. 
Recently, there is an intensive accumulation of information on the influence of various factors on the stress-strain rocks around the well and the improvement of research methods [6]. Methods of research of rocks around deep and superdeep wells can be divided into analytical, numerical, methods of physical modeling. Methods for studying the stress-strain state proposed by I.K. Fomenko, include a generalization of the experience of mathematical modeling of arrays of anisotropic rocks and the use of one of the numerical methods for solving problems of the theory of elasticity of anisotropic media - the method of boundary elements. In the general case, to determine the stresses in the selected mountain massif, there are known cases of using the finite element method under specific boundary conditions, such as the software package ANSYS and COMSOL Multiphisics [4]. Global stresses of the mountain massif make significant changes in the distribution of local stresses near the vertical deepest well (about $8000 \mathrm{~m}$ ). At the same time, the author proved that only classical calculation methods are sufficient for the preliminary assessment of the stress-strain state on the well wall.

Analysis of the current state of the problem allows us to draw the following conclusions:

1. The problem of predicting the stress-strain state of deep wells is quite relevant and widely studied, but the use of classical analytical approaches solves the problem only for certain partial cases.

2. The use of phenomenological approaches with the use of generalized parameters for assessing the state of deep wells requires the presence of objective, uncorrected input actual geological and physical information, which is not always possible.

3. Satisfactory results of predicting the stress-strain state of wells can be obtained using the finite element method in already tested software in combination with classical calculations and physical modeling, but solving the problem in this way requires appropriate scientific level of specialists. The issues of estimating the stress-strain state of deep wells remain incompletely studied and require certain engineering techniques for rapid assessment of the state of the well.

\subsection{The method of the analysis of elastic - plastic defor- mations of a deep well and a zone around a well is offered \\ The problem was solved in the following formulation (close to the problem of YM Lieberman) [2,4]: the stress state of a heavy iso-}


tropic, homogeneous elastic-plastic massif near the vertical cylindrical development of the final depth in this massif was studied. The elementary section (element) of a deep well was considered as an element with a hole of constant cross-section with a radius having an internal pressure $P_{1}$ and an external radius and external pressure $P_{2}$. The solution investigated a mathematical model that includes the equations of equilibrium, the relationship between the components of deformation and the components of displacement. Equations describing the properties of the medium, stress intensity and strain intensity are studied. The following boundary conditions are accepted: on the walls of the well evenly distributed stresses $\sigma_{r}=-P_{1}$; at the boundary of elastic and inelastic deformations, the continuity of the values of stresses and strains is observed.

The calculation is based on the assumption of uniform load distribution over the thickness of the element and the absence of loads in the planes parallel to the longitudinal axis of the element. The problem is axisymmetric, because weight is the only external force acting on the array, and deformation movements in the considered halfspace with a vertical cylindrical circular work take place only in the planes passing through the axis of symmetry. In all such planes the distribution of deformations and stresses will be the same. From the accepted assumptions it follows that the stress state of all points of the element is flat. This allows to reduce the solution of the volume problem to the solution of the distribution of deformations and stresses in one plane passing through the axis of symmetry of the well (to a flat deformed state).

The assumption is valid if $\left(r_{2} / t\right) \geq 2$ (where $t$ is the thickness of the element of the deformed part of the array). From the condition of load symmetry it can be assumed that stresses, deformations and displacements are functions of the radius and mechanical properties of the array material. When deriving the calculated equations, the influence of well contour irregularities, which lead to the concentration of local stresses, was neglected. The calculation assumed that the tensile diagram of the array material has no reinforcement. Depending on the external influence and the state of mechanical properties of rocks of the considered element of a deep well and physicochemical properties of clay solution used for drilling (flushing fluid), its most loaded points may be points located on the inner contour of the well. 
There begin to occur plastic deformations, ie the plastic region is adjacent to the inner contour of the well. The radius of the boundary of the plastic and elastic regions is marked (Fig. 3).

To derive the basic equation of equilibrium of the investigated element of a deep well by circumferential and radial stresses $\sigma_{r}$, their action on the average radius of the element $(r+d r / 2)$ is considered [8].

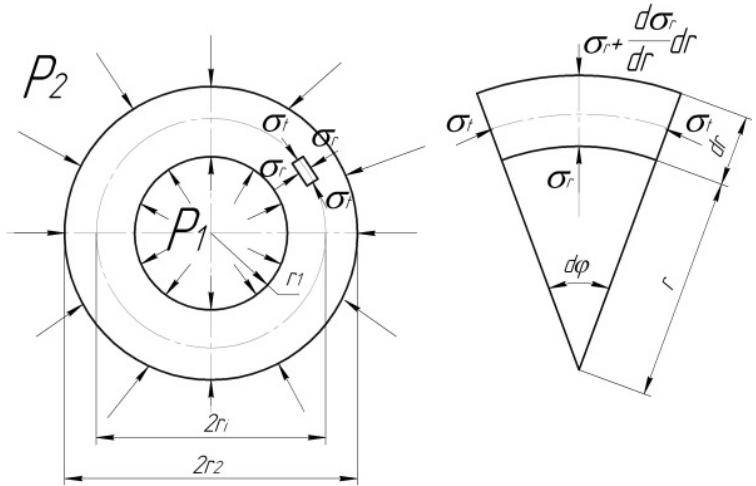

Fig. 3. Stress in the investigated element of the deep well

For a face at a distance away from the center of the element $r+d r$, the stresses will be equal to

$$
\sigma_{r}+\frac{d \sigma_{r}}{d r} d r
$$

To write the relationship between $\sigma_{t}$ and $\sigma_{r}$ design all the forces acting on the element in the direction of the mean radius we obtain

$$
\sigma_{r} r d \varphi+2 \sigma_{t} d r \sin \frac{d \varphi}{2}-\left(\sigma_{r}+\frac{d \sigma_{r}}{d r} d r\right)(r+d r) d \varphi=0 .
$$

Given that $\sin d \varphi / 2 \approx d \varphi / 2$ and neglecting the members of the higher order of smallness, the equation of equilibrium can be represented as follows

$$
r \frac{d \sigma_{r}}{d r}+\sigma_{r}-\sigma_{t}=0 .
$$

In the resulting equation there are two unknown stresses $\sigma_{t}$ and $\sigma_{r}$. To determine them, it is necessary to derive another equation, taking into account the boundary conditions of the problem:

1. On the contour of the well and the considered array of stress $\sigma_{r}=-P_{1}\left(P_{2}\right)$; 
2. On the boundary of elastic and non-elastic deformations of the array (at $r=r_{c}$ ) the equality of stresses is observed $\sigma_{r}^{e l}=\sigma_{r}^{p l}$ and $\sigma_{t}^{e l}=\sigma_{t}^{p l}$ displacement $u^{e l}=u^{p l}$, where $P_{1}$ is the back pressure in the well, $P_{2}$ is the lateral component of the gravitational forces acting on the formation, $r$ is the current value of the radius in the considered zone of the well. Due to the peculiarities of the problem, where the elastic-plastic massif is considered, plastic, elastic-plastic and elastic deformations can take place in the wellbore zone. Therefore, the plastic and elastic zones of the loaded massif with the well were investigated. The values of stresses at which plastic deformations appear on the inner surfaces of the well are found from the Mises plasticity condition [7]

$$
\left(\sigma_{r}-\sigma_{z}\right)^{2}+\left(\sigma_{t}-\sigma_{z}\right)^{2}+\left(\sigma_{r}-\sigma_{t}\right)^{2}=2 \sigma_{T}^{2} .
$$

Since normal stresses are not considered in the element (they are assumed to be evenly distributed throughout the array), the equation can be represented as (equivalent stresses of the energy hypothesis of plastic deformations)

$$
\sigma_{t}^{2}-\sigma_{t} \sigma_{r}+\sigma_{r}^{2}=\sigma_{T}^{2}
$$

where $\sigma_{T}$ - the yield strength of the material of the well element at the considered depth.

\section{Stress in the plastic zone.}

Using the known dependences [4,7], to identically satisfy the last equation, we introduce the function $\varphi$, with which we write $\sigma_{t}$ and $\sigma_{r}$ in the plastic stress region as follows

$$
\sigma_{t}=\frac{2}{\sqrt{3}} \sigma_{T} \cos \left(\varphi-\frac{\pi}{6}\right) ; \sigma_{r}=\frac{2}{\sqrt{3}} \sigma_{T} \cos \left(\varphi+\frac{\pi}{6}\right) .
$$

We enter dimensionless values of radiuses

$$
q=\frac{r}{r_{1}} ; \quad \beta=\frac{r_{c}}{r_{1}} ; \quad \alpha=\frac{r_{2}}{r_{1}} .
$$

Substituting the values of the circumferential and radial stresses (9) into equation (8), after the transformation we obtain the differential equation for the function $\varphi$

$$
q \frac{d \varphi}{d q}+\frac{\sin \varphi}{\sin \left(\varphi+\frac{\pi}{6}\right)}=0
$$

After integration we get 


$$
q=\frac{C(\sin \varphi)^{-\frac{1}{2}}}{e^{\frac{\sqrt{3}}{2} \varphi}}
$$

The constant integration $\mathrm{C}$ can be expressed in terms of the value of the function on the inner radius of the condition: when, $q=1, \varphi=\varphi_{1}$ and therefore.

Substituting the value of $C$, we obtain

$$
q=e^{\frac{\sqrt{3}}{2}\left(\varphi_{1}-\varphi\right)} \sqrt{\frac{\sin \varphi_{1}}{\sin \varphi}}
$$

Let us denote the value of $\varphi$ at the boundary of the elastic and plastic regions (for $r=r_{c}, q=\beta$ ) by $\varphi_{c}$. From equation (13) we have

$$
\beta=e^{\frac{\sqrt{3}}{2}\left(\varphi_{1}-\varphi_{c}\right)} \sqrt{\frac{\sin \varphi_{1}}{\sin \varphi_{c}}}
$$

To find the function $\varphi_{1}$, we use the boundary condition of the problem: $r=r_{l}$, for $q=1, \sigma_{r}=-P_{1}$. Then, given expression (9), we find

$$
-P_{1}=\frac{2}{\sqrt{3}} \sigma_{T} \cos \left(\varphi_{1}+\frac{\pi}{6}\right) .
$$

The obtained dependences (9), (14) and (15) allow to determine $\sigma_{t}$ and $\sigma_{r}$ in the plastic region.

\section{Stress in the elastic zone.}

Formulas for calculating stresses in the elastic region have the form [11]

$$
\sigma_{t}=\frac{2}{\sqrt{3}} \sigma_{T}\left(c_{1}+\frac{c_{2}}{q^{2}}\right) ; \sigma_{r}=\frac{2}{\sqrt{3}} \sigma_{T}\left(c_{1}-\frac{c_{2}}{q^{2}}\right) ;
$$

Using the condition of continuity $\sigma_{t}$ and $\sigma_{r}$ at the boundary of elasticity and plastic regions, for formulas (13) and (14) we take

$$
\begin{gathered}
\varphi=\varphi c, q=\beta \\
\cos \left(\varphi_{c}-\frac{\pi}{6}\right)=C_{1}+C_{2} \beta^{-2} ; \cos \left(\varphi_{c}+\frac{\pi}{6}\right)=C_{1}-C_{2} \beta^{-2}
\end{gathered}
$$

Solving these equations with respect to $C_{1}$ and $C_{2}$, we find

$$
C_{1}=\frac{\sqrt{3}}{2} \cos \varphi_{c} ; C_{2}=\frac{\beta^{2}}{2} \sin \varphi_{c}
$$

Substituting the values $C_{1}$ and $C_{2}$ found in (17), we obtain the dependences for stresses in the elastic region

$$
\sigma_{t}=\sigma_{T}\left(\cos \varphi_{c}+\frac{\beta^{2}}{\sqrt{3}} \frac{\sin \varphi_{c}}{q^{2}}\right) ; \sigma_{r}=\sigma_{T}\left(\cos \varphi_{c}-\frac{\beta^{2}}{\sqrt{3}} \frac{\sin \varphi_{c}}{q^{2}}\right)
$$


To find the value we use the boundary condition of the problem: when $r=r_{2}$, for $q=\alpha, \sigma_{r}=-P_{2}$

To find the value $\varphi_{c}$ we use the boundary condition of the problem: when we obtain

$$
-\frac{P_{2}}{\sigma_{T}}=\cos \varphi_{c}-\frac{e^{\sqrt{3}\left(\varphi_{1}-\varphi_{c}\right)}}{\alpha^{2} \sqrt{3}} \sin \varphi_{1}
$$

The calculation $\sigma_{t}$ and $\sigma_{r}$ is performed as follows: by formula (14) is the dimensionless radius $\beta$ of the boundary of the elastic and plastic regions; by formula (17) the values $\varphi_{c}$ are calculated; by formula (13) $-\varphi$, and from equations (19) the value of $\sigma_{t}$ and $\sigma_{r}$ is calculated.

\section{Radial displacements}

In solving the problem, the assumptions of the theory of plasticity about the incompressibility of the material both outside and within the elasticity are used. The incompressible material is characterized by the coincidence of the graphs of the dependence of the stress intensity on the intensity of deformations $\varepsilon_{i}$ with the tensile diagram. To calculate the radial displacement in the plastic region, we use the dependences of deformations on stresses $\sigma_{i}$ outside the elasticity at the biaxial stress state of incompressible material [11,12]

$$
\varepsilon_{t}=\frac{\varepsilon_{i}}{2 \sigma_{i}}\left(2 \sigma_{t}-\sigma_{r}\right) ; \varepsilon_{r}=\frac{\varepsilon_{i}}{2 \sigma_{i}}\left(2 \sigma_{r}-\sigma_{t}\right)
$$

$\varepsilon_{r}$ and $\varepsilon_{t}$ - respectively radial and circumferential relative deformation, $\varepsilon_{t}$ - intensity of deformation, $\varepsilon_{i}=\frac{2}{\sqrt{3}} \sqrt{\varepsilon_{t}^{2}+\varepsilon_{r}^{2}+\varepsilon_{i} \cdot \varepsilon_{r}}$. Dividing $\varepsilon_{r}$ by $\varepsilon_{t}$ we get

$$
\frac{\varepsilon_{r}}{\varepsilon_{t}}=\frac{2 \sigma_{r}-\sigma_{t}}{2 \sigma_{t}-\sigma_{r}}
$$

Circular $\varepsilon_{t}$ and radial $\varepsilon_{r}$ deformations are associated with radial displacement and dependencies [12]

$$
\begin{aligned}
& \varepsilon_{t}=\frac{\nu}{r} \text { and } \varepsilon_{r}=\frac{d \nu}{d r} \\
& \varepsilon_{i}=v / r a n d \varepsilon_{i}=d v / d r
\end{aligned}
$$

Substituting the obtained values is represented, as 


$$
\frac{d v}{v}=\frac{2 \sigma_{r}-\sigma_{t}}{2 \sigma_{t}-\sigma_{r}} \cdot \frac{d q}{q}
$$

Differentiating $q$ we obtain

$$
\frac{d q}{q}=-\frac{1}{2}(\sqrt{3}+\operatorname{ctg} \varphi) d \varphi .
$$

Substituting expressions into formula (24), we obtain

$$
\frac{d v}{v}=\frac{\sin \left(\varphi-\frac{\pi}{6}\right)}{\sin \varphi} d \varphi
$$

After integration, we write the equation for finding the radial displacement in the plastic zone.

$$
\frac{v}{r_{1}}=\frac{\sigma_{T}}{2 E} C_{3} \frac{e^{\frac{\sqrt{3}}{2} \varphi}}{\sqrt{\sin \varphi}},
$$

where $E$ is the modulus of elasticity. Formula for radial movement in the elastic zone for incompressible material using Hooke's law will be

$$
u=\varepsilon_{t} r=\frac{r_{1}}{2 E}\left(2 \sigma_{t}-\sigma_{r}\right) q
$$

Substituting the value $\sigma_{t}$ and $\sigma_{r}$ into the obtained expression, we obtain the equation of radial displacements in the elastic zone during elastic-plastic deformation

$$
\frac{u}{r_{1}}=\frac{\sigma_{T}}{2 E}\left(q \cos \varphi_{c}+\frac{\sqrt{3} \beta^{2} \sin \varphi_{c}}{q}\right)
$$

From the condition of equality of radial displacements at the boundary of the plastic and elastic regions, it is possible to determine the constant integration of $C_{3}$ by equating radial displacements by formulas (26) and (28) when $q=\beta, \varphi=\varphi_{c}$

$$
C_{3}=\frac{2 \beta \sqrt{\sin \varphi_{c}} \sin \left(\varphi_{c}+\frac{\pi}{6}\right)}{e^{\frac{\sqrt{3}}{2} \varphi_{c}}}
$$

Then the equation of radial displacements in the plastic zone will be 


$$
\frac{u}{r_{1}}=\frac{\sigma_{T}}{E} \beta e^{\frac{\sqrt{3}}{2}\left(\varphi-\varphi_{c}\right)} \sqrt{\frac{\sin \varphi_{c}}{\sin \varphi}} \sin \left(\varphi_{c}+\frac{\pi}{6}\right)
$$

\section{The order of calculation.}

For a given pressure $P_{1}$ from relation (17) are the value $\varphi_{1}$, and for a given value $P_{2}$ from formula (21) find $\varphi_{\mathrm{c}}$. After that, from relation (16) get $\beta$. Stresses in the plastic region are calculated by formulas (9), and in the elastic region by equations (20).

The transition from $\varphi$ to the dimensionless radius $q$ in the plastic zone is carried out by expression (15). Radial displacements in the plastic and elastic regions are calculated by equations (30) and (28), respectively.

The stress field formed in the shaft zone differs from the initial concentration of stresses in the well walls, resulting in elastic and inelastic movements of rocks. The above method is valid only in the presence of the latter, in other cases, the assessment of the stressstrain state of rocks can be performed using the following equations

$$
\sigma_{t}=\frac{P_{1}-P_{2} \alpha^{2}}{\alpha^{2}-1}+\frac{\left(P_{1}-P_{2}\right) \alpha^{2}}{\left(\alpha^{2}-1\right) q^{2}} ; \quad \sigma_{r}=\frac{P_{1}-P_{2} \alpha^{2}}{\alpha^{2}-1}-\frac{\left(P_{1}-P_{2}\right) \alpha^{2}}{\left(\alpha^{2}-1\right) q^{2}}
$$

Radial displacements during elastic deformations of an element of incompressible material can be found by the formula obtained after substitution (31) in (27)

$$
v=\frac{r_{1}}{E\left(\alpha^{2}-1\right)}\left[(1+\mu)\left(P_{1}-P_{2}\right) \alpha^{2}+(1-\mu)\left(P_{1}-P_{2} \alpha^{2}\right) q^{2}\right] \frac{1}{q}
$$

The recommended calculation method allows to assess the stressstrain state of the deep wellbore on a sufficient (for engineering practice) scientific basis. And also the method allows to formulate, in particular, requirements concerning stability of a well. It should be borne in mind that it is very difficult to eliminate the inelastic deformation of the well walls, which has already begun, and sometimes it is simply impossible to stop it. It is much more effective in such cases to prevent the possibility of violating the integrity of the array, and for this it is necessary to pre-establish its stress state. In order to formulate the strength condition (the condition of plastic deformations at the point), the strength criterion is introduced into the calculation. 
Assuming that the well element is exposed to evenly distributed internal and external pressures, the deformations will be symmetrical about the longitudinal axis of the element, then the stress intensity (based on the Mises plasticity condition) - a generalized strength condition will be

$$
\left[\sigma_{i T}\right] \geq \frac{1}{2} \sqrt{\left(\sigma_{r}-\sigma_{z}\right)^{2}+\left(\sigma_{z}-\sigma_{t}\right)^{2}+\left(\sigma_{r}-\sigma_{t}\right)^{2}}=\sigma_{i p a c},
$$

where $\sigma_{\mathrm{ipac}}$ - the calculated value of stresses $\sigma_{\mathrm{i}}$; [ $\left.\sigma_{\mathrm{i}}\right]$ - intensity of stresses at which plastic deformations appear in the rocks of the massif at the studied depth of the well. The criterion of strength does not contain any elastic constants. The condition of transition of material from elastic state to plastic beyond the yield strength is accepted, ie the condition of plasticity (if the material is able to accept plastic deformations without collapsing).

It is known $[13,25]$ that the plastic state or destruction of the material occurs when a certain ratio between octahedral and normal stresses is satisfied

$$
\sigma_{0}=\frac{1}{3}\left(\sigma_{z}+\sigma_{r}+\sigma_{t}\right) \leq\left[\sigma_{T}\right],
$$

where $\sigma_{0}$ - hydrostatic (average normal) pressure at the point. It does not depend on the choice of coordinate axes, ie it is invariant. In addition, it should be borne in mind that the hydrodynamic fluctuations of pressure in the well also cause changes in the stress state of the rocks of the array at the considered depth of the well. The pressure in the well increases above the hydrostatic $P_{1}$ during the operation of the pumps during drilling due to hydraulic resistance of the fluid pressure and during the descent of the tool due to the compression of the fluid. When lifting the tool, the pressure decreases due to the piston effect, as well as when the fluid level in the well decreases. The change in the stress state of rocks in this case can be determined as follows $\Delta \sigma_{z}=0$ since the change in hydrostatic pressure does not affect the geostatic pressure $P$

$$
\begin{array}{lc}
\sigma_{r}=\sigma_{r}-\left[\left(\lambda P_{r}-P_{1}\right) \frac{r_{1}^{2}}{r^{2}}-\lambda P_{r}\right] & \lambda=\frac{\mu}{1-\mu} ; \\
\sigma_{t}=\sigma_{r}+\left[\left(\lambda P_{r}-P_{1}\right) \frac{r_{1}^{2}}{r^{2}}-\lambda P_{r}\right] & P_{1}=\gamma_{\rtimes} H \pm P,
\end{array}
$$


where $\gamma$ - specific weight of flushing fluid, $\Delta \mathrm{P}$ - dynamic change of fluid pressure in the well. Using the principle of independence of forces, add the stresses due to various factors, and get

$$
\Sigma \sigma_{z}=\sigma_{z} ; \Sigma \sigma_{r}=\sigma_{r}+\square \sigma_{r} ; \sigma_{t}=\sigma_{t}+\square \sigma_{t}
$$

In this case, the strength condition in the cylindrical coordinate system will be

$$
\sigma_{i s} \geq \frac{1}{2} \sqrt{\left(\sum \sigma_{z}-\sum \sigma_{r}\right)^{2}+\left(\sum \sigma_{z}-\sum \sigma_{t}\right)^{2}+\left(\sum \sigma_{r}-\sum \sigma_{t}\right)^{2}} \leq\left[\sigma_{T}\right]_{(37)}
$$

According to experimental data, the long-term strength of rocks in deep wells is $0.85-0.95$ of its original value, ie: $\sigma_{i s_{p a c}}=(0,85-0,95) \sigma_{i s}$

\subsection{Interpretation of the results of calculations of the stress- strain state of the wellbore and wellbore}

Drilling a well significantly changes the stress-strain state of the elastic and elastic-plastic massif. In the shaft zone, in comparison with the massif located outside the boundary of the well, the rocks undergo a qualitatively new stress-strain state even in the presence of rocks prone to elastic-plastic deformation. The components of the stress tensor in this region at certain values of the external forces $\left(P_{r}, P_{1}, P_{2}\right)$ can have both positive (tensile) and negative (compressive) values (Fig.4).

Rocks in this interval (depth $3935 \mathrm{~m}$ in the well № 230 Borysivska Square) are in a difficult stressful state. They are simultaneously exposed to normal compressive $\sigma_{r} \cdot \sigma_{z}$ and tensile stresses $\sigma_{\mathrm{t}}$. Quite high compressive normal stresses are distributed along the trunk walls. They exceed the values of compressive normal stresses of the rock (1.0-0.8 times) and the maximum value of tensile stresses $+20.0 \mathrm{MPa}$. 


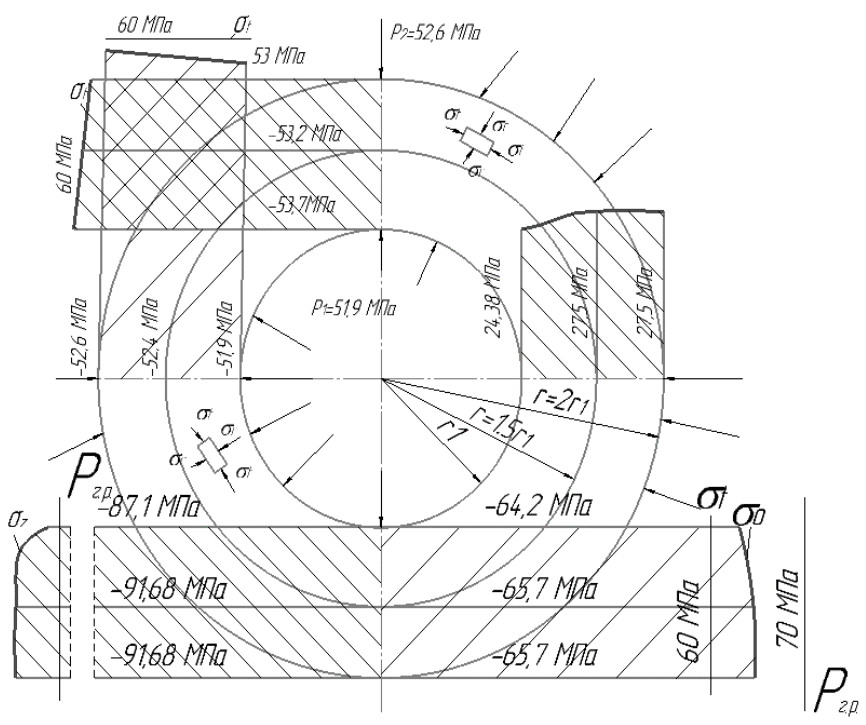

Fig. 4. Quasi equilibrium stress distribution along the well cross section

The intensity of stresses on the well circuit also has a maximum value $\sigma_{\mathrm{T}}$ exceeding 1.1 times. Only stresses $\sigma_{0}$ are minimal here, their valuesare almost $0.5 \sigma_{T}$

For $\sigma_{T}$ the accepted conditional yield strength, which characterizes the allowable amount of stresses that cause plastic deformation of the rock in terms of its occurrence. On the horizontal plane (when passing the array) near the wellbore, all stresses change their magnitude. At the boundary of the array, represented by a surface with radiuses $r_{1}$ and $r_{2}$, only compressive normal stresses act. Here $\sigma_{\mathrm{z}}$ they are growing rapidly at the level of reaching their limits.

In this area, the stresses $\sigma_{t}$ with a large gradient decrease, change the sign to the opposite, and again with a large gradient begin to increase. At the boundary where $r=r_{2}$ the stress has positive values $\left(\sigma_{t}\right.$ $=+3.94 \mathrm{MPa}$ ). Stresses $\sigma_{i}$ with approximately the same intensity $\sigma_{t}$ without jumps and differences are sharply reduced. Of all the calculated compressive stresses on the surface, which is limited by radiuses $r=r_{1}$ and $r=r_{2}$, only $\sigma_{0}$ have minimal values.

When passing through the section from $r_{1}$ to $r_{2}$ the absolute value of these stresses increases according to the linear law. Next, the cross 
section into the depth of the array, all components of the stress tensor remain compressive: $\sigma_{t}$ they increase intensively and reach their maximum value at: $r=1.5 r_{1}$ slightly decrease, then increase to the value of lateral pressure $\left(\sigma_{r}=-P_{2}=-52,6 \mathrm{MPa}\right)$.

Remain constant and equal (in absolute value) to geostatic pressure. The magnitude of stresses $\sigma_{i}$ decreases to its minimum values. Further removal of the wellbore from the cross section into the depth of the massif indicates that all stresses $\left(\sigma_{r}, \sigma_{t}, \sigma_{0}, \sigma_{z}, \sigma_{i}\right)$ are close to their constant values, Here the rocks are in a state of stable equilibrium, close to the state of comprehensive compression (Fig. 4). The magnitude of the applied stresses, determined by this state, also exceeds the yield strength of rocks. But it is below the values corresponding to fracturing.

The hydrostatic pressure in this case has a maximum value of approximately $66 \mathrm{MPa}$. That is, the rocks in the intact massif are in a state of elastic-plastic deformation. The instantaneous radial displacements of the loaded rock enclosed in the zone of influence of the well on the array and prone to elastic-plastic deformations are (1.5-1.3) $\mathrm{mm}$. The values of stresses outside the zone of influence of the well on the rock mass are: $\sigma_{r}=-52,6 \mathrm{MPa} ; \sigma_{z}=-91,68 \mathrm{MPa} ; \sigma_{t}=-$ $56,2 \mathrm{MPa} ; \sigma_{i}=26,4 \mathrm{MPa}$ and $\sigma_{0}=-66,8 \mathrm{MPa}$.

Here the rocks are in a state of stable equilibrium, close to the state of comprehensive compression. As can be seen from the diagram in Fig. 4, the value of the applied stresses, determined by this state, also exceeds the value of the yield strength of rocks, but below their fracturing. The hydrostatic pressure in this case has a maximum value of approximately $66 \mathrm{MPa}$. That is, the rocks in the intact array are in a state of elastic - plastic deformation. The instantaneous radial displacements of the loaded rock enclosed in the impact zone of the well are $1.5-1.3 \mathrm{~mm}$.

For the case under consideration, the size of the zone of influence of the well on the rock mass is limited to the surface with a radius $r=1.5 r_{l}$. Outside this zone there is a stress state due to gravitational forces. There is a quasi-equilibrium state of rocks (under the influence of comprehensive non-uniform compression) is prone to impaired stability in cases of additional influence or degree of freedom. The appearance of an additional degree of freedom will negatively affect the state of equilibrium. This will cause a sharp redistribution 
of stresses, destruction of rocks or their creep due to plastic deformation. Deformation can occur in almost any direction.

A number of researchers based on the analysis of experimental data stated a pattern. At loading of rock and its endurance under loading within 8-10 hours there is a change of a stressful condition of breed. In this case, there is a relaxation of stresses. In the future, the process stabilizes. The stress-strain state of rocks remains virtually unchanged. The most intense reduction of stresses is observed in the trunk zone and in the initial period after loading (weakening). Analysis of the values of relaxation and design stresses showed a decrease in their time by $5-15 \%$ (in clay rocks up to $10 \%$ ).

The effect of relaxation on the magnitude of stresses found using the proposed method can be taken into account by introducing correction factors $K_{\mathrm{p}}$ and $K_{\mathrm{m}}$. They take into account the relaxation of stresses in the shaft zone and outside the impact of the well on the rock mass $\left(K_{\mathrm{p}}=0.9-0.85 ; K_{m}=0.9-0.95\right)$. When quantifying the values of operating stresses in rocks, it is necessary to take into account another feature. In rock in the conditions of its natural occurrence both properties of breed, and values of its mechanical characteristics change in due course. Experimental and theoretical studies have shown a decrease in allowable stress values, ie their change according to the law of long-term strength of rocks in well conditions is $0.85-0.95$ from the initial [12].

Taking into account the above two features in the behavior of rocks in terms of their occurrence, we can assume that the stress state of rocks during well drilling changes over time in proportion to changes in the values of rock strength characteristics under the condition of their long strength. The decrease in the strength of rocks in the well according to the law of long-term strength is compensated by the relaxation of the applied stresses. At final comparison of results of calculation (quantitative estimation of settlement data $\sigma_{T}$ ) it is necessary to enter a correction factor. It takes into account the discrepancy of the values of $\sigma$ obtained by the calculation method with the real ones, due to stress relaxation and long-term rock strength. The values of this coefficient are in the range of $0.85-0.95$

The rock mass is considered as an elastic medium in which the well is drilled. In this environment under the influence of loads there is a creep of deformations and relaxation of stresses. The wellbore is 
deformed inward by some amount. After continuing to deepen the well, the process of contour movements continues due to the creep of deformations, but it is opposed by the resistance of the wellbore and the pressure of the drilling fluid. Their joint deformation continues until the state of equilibrium is realized in the system "wellbore rock". Deformation is characterized by a fairly rapid process and a sharp increase in displacement after they reach a certain critical limit in the previous area - the limit of elasticity. This circumstance is usually repeated, which in itself suggests that the cause of this pressure is some general physical pattern. Its characteristic feature is the very rapid growth of deformations in a relatively short period of time, which occurs after the plastic deformations reach some critical limits.

From the energy point of view, the following happens: with a slight change in the stress state associated with the deformation of the face, there is a relatively rapid release of potential energy spent on additional destruction of structural bonds in the rock mass under tensile stresses $\sigma_{t}$. This is accompanied by a significant increase in volume (landslides, falling into the well). After that, the mechanical system finds a state of equilibrium again. The rate of transition from one energy state to another is determined by the stress state and structural features of rocks in the array. For fragile rocks, it can be very high. For rocks containing a large number of clay particles, the transition rate will be relatively small, and the subsequent process will be long (Fig. 4). In this phenomenon - is the elastic-plastic loss of stability of the equilibrium of the rock mass (wellbore). When drilling a well in the bottomhole zone, the level of stresses in the array (on the walls of the well) changes due to the appearance of caverns of different sizes.

Sometimes fragile layers, not of great power, lie directly above the drilled layer. This layer is destroyed, as a rule, immediately after the drilling of the formation and its retention with the solution is very difficult. Crashing occurs in small areas along the wellbore. The nature of the direct profile of the well in this case is determined by the capacity of the destroyed rocks after the transfer of the drilling process into the well. The time of involuntary formation of the profile of the wellbore is several times less than the time of formation of the main profile. 
At the time of the first collapse of the wellbore there is a rapid change in the nature and magnitude of the loads directly on the wellbore. During this period, there may be a large deformation of the trunk, which sometimes leads to complete destruction of the well. After the first collapse, the thickness of the weakened rocks of the wellbore decreases, the stress level in the bottomhole part of the rock mass decreases and the operating conditions of the wellbore improve. The wellbore, in this case with a cavity, may not be fixed for a long time. This sometimes allows you to go through the whole layer without significant complications. To control the condition of the wellbore and the zone of impact of the well, rock pressure $P_{2}$ and hydrostatic pressure $P_{1}$ in deep wells provide, taking into account the stress-strain state of the wellbore and stump zone of the rock mass, change the drilling fluid density $\gamma$. The same role is performed by works related to the change of stresses acting on the wellbore and the wellbore zone.

The rock mass before the extraction of the rock is in an equilibrium stress-strain state, which is called the initial. Stress components that can be caused by neotectonic processes are usually considered absent. As the well deepens and the bottom moves, the stress field around the well changes. The area of the rock mass within which these changes occur is called the impact zone of the well. This zone of influence of the well is small, but often the deformation processes affect the entire thickness of the drilled formation (Fig. 4). There are two characteristic areas around the well. The area within which the voltages are less than the initial ones is called the discharge zone. The area within which stresses in excess of the initial are called the reference pressure zone. As the bottom of the well moves in space, the selected areas also move with it. In this case, the rocks of the array, according to changes in the stress-strain state, can gradually move from one zone to another. Under the influence of significant stresses of the rock in the shaft part of the formation, located close to the wellbore, are destroyed. Their bearing capacity decreases and the maximum stress moves deep into the array. The destroyed part of the rocks is intensively pressed into the well (cavern formation). The parameters of this well zone depend on many factors: determining the depth of the well, the ratio of the components of the initial stress field, the size and configuration of the impact zone of the well on the 
rock mass, deformation and strength properties of rocks, their structure and texture.

When calculating the stress-strain state of the walls of deep wells, considerable attention should be paid to the coefficient of strength, its justification. with Depending on the tasks, its values are taken equal to 1.8-3.0. The fracture pressure value is calculated as the difference between the downhole pressure during fracturing and the crack closing pressure. In this regard, the problem of estimating the strength of the unfixed part of the formation is important for establishing the stress-strain state of the shaft and the shaft zone of a deep well. The stability of an unfixed well is assessed by the absence of dumps, destruction of rocks in its walls, debris.

The study of mechanical processes in the wellbore massif of rocks is carried out on the basis of the above general principles of mechanics of continuous media. Although these processes in the well and in the rocks of the formation are a whole, the features of the stress-strain state in these elements have significant differences; in the massif outside the zone of influence of the well, the rocks work on compression and can be destroyed by crushing. In the walls of the well and in the massif of the impact zone of the rock are bending deformations, more dangerous in terms of destruction. Therefore, to assess the stability of the unfixed part of the well, it is advisable to consider separately the strength of the rocks of the shaft part of the array, the strength of the rocks of the wellbore.

The proposed method of calculation allows to find the values of radial and tangential stresses and displacements and the radius of the region of inelastic deformations of rocks. We can determine the displacements that occur both on the walls of wells and in adjacent areas, in non-elastic and elastic zones. The method allows you to easily, quickly and reliably establish the stress state of the rocks that make up the walls of the well, as well as near it. After that, make objective decisions when drilling wells under specified conditions. Thus, to 
ensure a reduction in material and labor costs, especially in the arrangement of oil and gas fields, should take into account the state of the mountain pores. The state of rocks is determined by the action of gravitational and tectonic forces. This allows you to make the recommended engineering method of calculation.

\section{Conclusions}

The method is recommended for quick decision-making related to various problems that arise during the construction of deep wells (oil, gas, etc.) and their operation. It can also be used in the design and implementation of analytical research. For engineering calculations of wells in a narrow range of stresses, the elastic and strength parameters of the rock can be considered constant. Thus, the known laws of elasticity and plasticity were used to determine deformations and stresses.

Therefore, the values of the instantaneous (calculated characteristics of the rocks by values) and the ratio of the applied stresses, taking into account the long-term strength will be approximately the same and equal to about one. Therefore, the quantitative assessment of the stress state of the rocks along the wellbore and near the wellbore with an accuracy sufficient for engineering practice, can be performed using the proposed method.

\section{References}

1. Alimzhanov, A.M. (1994). Stress-strain state around deep wells in conditions of heterogeneity of mechanical properties of rocks near the wellbore zone: Ph.D. dis. for the scientific degree Physics and Mathematics, 01.02.04 Mechanics of a Deformable Solid Body. Novosibirsk. $-32 \mathrm{p}$.

2. Alimzhanov, A.M. Spatial axisymmetric stability of a vertical well in an array containing a layer with reduced strength properties [Electronic resource] / A.M. Alimzhanov. - Access mode: http://www.ogbus.ru/authors/Alimzhanov/ Alimzhanov_1.pdf

3. Bandurina, O.V. (2012) Perspective of analysis of the stress-strain state of a deep wellbore // Abstracts of the 64th scientific conference of professors, academics, scientific practitioners, graduate students and students of the university. Volume 1. (Poltava, April 17 to May 11, 2012) - Poltava: PoltNTU, 2012. - PP 147 - 149. 
4. Blokhin, V.S. Bandurina, O.V. (2013) Assessment of the stress state of a deep wellbore // Bulletin of the KrNU named after Mikhail Ostrogradsky. -Vol 1/2013(78). - PP. 71 - 75.

5. Blokhin, V.S. Stability criteria for carbonate and terrigenous deposits in deep wells // Improving the efficiency of drilling deep wells in abnormal geological conditions / V.S. Blokhin, V.V. Ippolitov, V.D. Terentiev, F.K. Gimadeeva - Orenburg: TSNTI, 1983. - $44 \mathrm{p}$.

6. Blokhin, V.S. (1984) Method for assessing the stability of the well walls / V.S. Blokhin, V.V. Ippolitov, V.D. Terentiev // Oil industry. - 1984. - N. 7. - PP. $28-31$.

7. Investigation of the stress-strain state of the massif around the well with timevarying strength properties of rocks / P.I. Svitalka, V.V. Solovyov, M.P. Nesterenko, O.V. Bandurina // Scientific journal (Geology. Mining. Oil and gas business) / Poltava National Technical University named after Yuri Kondratyuk. - Vol. 1 (1). Poltava: PoltNTU, 2012. - PP. 144 - 148.

8. Eremenko, S.Yu. (1991) Finite element methods in the mechanics of deformable bodies / S.Yu. Eremenko. - Kharkov: Basis, 1991. - 272p.

9. Isakov, V.B. (2003) Elements of numerical methods: Textbook for students studying in the specialty Mathematics group Pedagogical special. - M.: Academy, 2003. -192 p.

10. Iudin, M.M. (1997) Stress-strain state of the frozen rock mass around a vertical shaft. - Yakutsk: YaGU, 1997. - 26 p.

11. Kalashnik, A.I., Kalashnik, N.A. (2010) Technogenic deformation of the bowels // Rational development of mineral resources, 2010. - №2. PP. 48 - 53.

12. Karev, V.I. (2010) Influence of the stress-strain state of rocks on the filtration process, and well flow rate: abstract of the thesis. dis. for the academic degree of doctor of technical sciences 01.02.04 - mechanics of a deformable solid body St. Petersburg. - 34 p.

13. Papusha, A.N., Gontarev, D.P. (2010) On the issue of calculating the stress-strain state of a rock mass in the vicinity of a superdeep vertical well // Visnik MSTU. - No. 5. - PP 81 - 93.

14. Svitalka, P.I. Solovyov, V.V. Bandurina, O.V. (2013) Methods and results of determining the mineral content of salty rocks fallow in terms of firmness of nonspring deformation //Collection of scientific practices: branch mechanical engineering. - Vol.3 (39). - PP.175 - 181.

15. Stasenko, V.M., Karpenko, V.M., Kozachenko, M.I. (2008) Problems of evaluation and forecasting of the geobaric and stress-strained mill of the mountain massif during the drilling of deep oil and gas sverdlovins // Oil and gas industry, 2008. - №. 2. - PP. 20 - 22.

16. Alimzhanov, M.T. (1992) Methods of continuum nonlinear mechanics in problems of rock pressure control at drilling deep wells / M.T. Alimzhanov // Advances in Mechanics, Int.Jour., Warsaw, 1992. - Vol.15. - № 3-4. - P. 41 - 69.

17. Gass R. (1998) Mathematica for scientists and engeneers: using Mathematica to do science/ monography — New Jersy «Prentice Hall», 1998 - 499 p. 